\title{
Review
}

\section{Immunohistochemical Classification of Skeletal Muscle Fibers}

\author{
Aiko Hori $^{1}$, Akihiko Ishihara ${ }^{2}$, Shigeo Kobayashi ${ }^{1}$ and Yasuhiko Ibata $^{3}$ \\ ${ }^{1}$ Department of Bio-informatics, Graduate School of Informatics, ${ }^{2}$ Laboratory of Neurochemistry, Faculty of \\ Integrated Human Studies, Kyoto University, Kyoto 606-8501 and ${ }^{3}$ Department of Anatomy and \\ Neurobiology, Kyoto Prefectural University of Medicine, Kyoto 602-0841
}

Received for publication May 11, 1998 and in revised form September 7, 1998

\begin{abstract}
Mammalian skeletal muscle fibers have several types of myosin heavy chain (MHC) isoforms. Immunohistochemical techniques enable us to identify the MHC isoform type in a single muscle fiber, i.e., $\mathrm{MHCl}$, MHClla, MHCIlx, or MHCIlb. In addition, hybrid single fibers having more than one MHC isoform type can be classified using immunohistochemistry. The presence of different MHC isoform(s) in a single fiber correlates

with the maximal contractile velocity and the enzyme histochemical reaction of the myofibrillar adenosine triphosphatase. The adaptive response of the skeletal muscle is the basis of the fiber-type transformation depending on the phenotypic expression pattern in several MHC isoforms. In this review article, we address the availability of the monoclonal antibodies which recognize specific MHC isoform(s) in a single fiber.
\end{abstract}

Key words: Enzyme histochemistry, Immunohistochemistry, Muscle fiber type, Myosin heavy chain isoform, Skeletal muscle

\section{Introduction}

Myosin heavy chain (MHC) in mammalian skeletal muscles has several types of isoforms [68, 69], which enables us to classify muscle fiber types depending on the MHC diversity. MHC isoforms of a muscle or a single fiber can be detected using electrophoresis or immunohistochemistry.

Immunohistochemistry has the advantage of being able to show the coexistence of multiple MHC isoforms within a single fiber in skeletal muscle. Although the regulation of muscle fiber-type transformation has not yet been clarified, immunohistochemical analyses will provide useful information for unsolved problems regarding muscle fiber-type plasticity. In this review article, we address the availability of the monoclonal antibodies which recognize specific $\mathrm{MHC}$ isoform(s) in a single fiber.

Correspondence to: Dr. Akihiko Ishihara, Laboratory of Neurochemistry, Faculty of Integrated Human Studies, Kyoto University, Sakyo-ku, Kyoto 606-8501, Japan.

\section{MHC Isoform of Muscle Fiber}

Myosin consists of six subunits; two heavy chains and two pairs of light chains. MHC is the major myofibrillar protein, and its adenosine triphosphatase (ATPase) activity is required for the contractile activity of muscle fiber. MHC protein is a product of a multigene family. At present, nine isoforms are identified as skeletal muscle MHC isoforms at both the protein and mRNA levels; MHCemb (embryonic), MHCneo (neonatal), MHCI (MHCI $\beta$ ), MHCIIa, MHCIIx (or MHCIId), MHCIIb, MHCeo (extraocular), MHCm (masticatory or mandibular), and $\mathrm{MHC} \alpha$ ( $\alpha$-cardiac-like) [19, 62, 69, 94]. Among them, the major isoforms in adult skeletal muscles are MHCI, MHCIIa, and MHCIIb. These MHC isoforms have been separated using electrophoretic techniques [21] and coded by three distinct genes [41]. Thereafter, a fourth MHC isoform in adult skeletal muscles called MHCIIx (or MHCIId) has been investigated [7, 48, 70]. There are also some reports regarding MHC isoform variants such as MHCIton (slow-tonic) [49] and MHCIa [26, 28], which have not yet been cloned.

Myosin forms bipolar thick filaments that interdigitate with thin actin filaments in the sarcomere, forming 
the contractile unit of muscle fiber. In the myosin head near the ATP- and actin-binding sites, there are two regions which are flexible in structure and variable in amino acid sequence $[6,75]$. These two regions in the motor domain of myosin are thought to produce the difference in the maximum velocity of the myosin molecule during movement along a single myosin head, which leads to the correlation between the ATPase activity and the speed of shortening in both the whole muscle [8] and the skinned muscle fiber $[13,15]$. Therefore, the presence of specific MHC isoform(s) is related to muscle fiber contractile properties, such that the maximal contractile velocities are in the rank order of MHCI $<$ MHCIIa $<$ MHCIIx $<$ MHCIIb [13]. Even in hybrid fibers in which multiple MHC isoforms coexist, the contractile property depends on the proportion of the MHC isoform types in the hybrid fiber [14]. Functional differences of MHC isoforms in skeletal muscle have been extensively investigated $[38,86]$.

Some other proteins such as myosin light chain, troponin, actin, sarcoplasmic (endoplasmic) reticulum $\mathrm{Ca}^{2+}$-ATPase (SERCA), and parvalbumin contribute to the identification of specific fiber types and have different isoform expressions between slow- and fast-twitch fibers $[56,69,71,74,91,95]$. The functional diversity of the skeletal muscle is, however, most adequately represented by the MHC isoform distribution of the muscle.

\section{Immunohistochemical Classification of Muscle Fiber}

Mammalian skeletal muscles are composed of heterogeneous types of fibers based on their different MHC isoform expressions. This heterogeneity is present in different muscles of the same species and in the same muscles of different species.

MHC isoforms expressed in a single fiber can be determined by immunohistochemical techniques. Monoclonal antibodies (MAbs) for the classification of the four major MHC isoform types (MHCI, MHCIIa, MHCIIx, and MHCIIb) are used for immunohistochemistry, and the specificity of the MAbs is either for a single MHC isoform or for multiple MHC isoforms. Prior to a study, however, the specificity of MAbs in the subject animal should be tested, because some MAbs have interspecies variability in their specificities.

Figure 1 shows serial transverse sections of the rat soleus muscle stained with different MAbs (panels A to D), as well as for ATPase (panels E to G) and succinate dehydrogenase (SDH) (panel $\mathrm{H}$ ) activities. The specificity of these MAbs for rat MHC isoforms is summarized in Table 1. Rat soleus muscle, which is a typical slow-twitch muscle, contains mainly MHCI and MHCIIa [22, 85], and the proportions of these types change with postnatal development [83]. In the adult rat, approximately $80 \%$ of the total fibers in the muscle are stained positively with MAb Slow (specific for MHCI), and those fibers are marked as 1 (panel A). The rest of the fibers are stained positively with both MAb Fast (specific for all types of MHCII) and MAb 71 (specific for MHCIIa), and marked as 2 in panels $B$ and $C$, respectively. None of the fibers are stained positively with MAb F3 (specific for MHCIIb, panel D), and all of the fibers are stained positively with MAb 35 (specific for all types of MHCs except MHCIIx, not shown). The staining pattern of each MAb is almost homogeneous, implying the diffuse distribution of MHC isoform molecules in the fiber cross-sectional area. The distribution of the MHC isoform along the fiber length is, however, reported to be nonuniform even in the skeletal muscle under normal conditions [80], and the nonuniformity increases under muscle fiber-type transformation [29, 80]. Note that two fibers marked as 3 are stained positively with MAbs Slow (panel A), Fast (panel B), and 71 (panel C), indicating that they are hybrid fibers character-

Table 1. Monoclonal antibody specificity

\begin{tabular}{lcccc}
\hline \multirow{2}{*}{ MAb } & \multicolumn{5}{c}{ MHC isoform } \\
\cline { 2 - 5 } & I & IIa & IIx & IIb \\
\hline Slow & + & - & - & - \\
Fast & - & + & + & + \\
71 & - & + & - & - \\
F3 & - & - & - & + \\
35 & + & + & - & + \\
D9 & - & - & + & + \\
N1.551 & - & ++ & $+/-$ & - \\
A4.1519 & - & ++ & - & $+/-$ \\
\hline
\end{tabular}

The specificity of each monoclonal antibody (MAb) bound to specific myosin heavy chain (MHC) isoforms in rat muscle fibers was determined by Bottinelli et al. [14] and Schiaffino et al. [70] and according to the instructions of the supplier. +, positive reaction for that MAb with that specific MHC isoform(s); - , no reaction between MAb and MHC isoform(s); ++ , positive reaction with higher intensity, $+/-$, positive reaction with lower intensity. The MAbs used in this study were types IgG (Slow, Fast, 71, and 35) and $\operatorname{IgM}(\mathrm{F} 3, \mathrm{D} 9, \mathrm{~N} 1.551$, and A4.1519).

Fig. 1. Serial transverse sections of the soleus muscle of an 8-week-old rat. The sections are stained with the specific monoclonal antibodies against specific myosin heavy chain (MHC) isoforms (panels A-D, see Table 1 for specificity) and for ATPase activities following preincubations at pH 10.3 (panel $\mathbf{E}$ ), 4.5 (panel $\mathbf{F}$ ) and 4.3 (panel $\mathbf{G}$ ) and for SDH activity (panel $\mathbf{H}$ ). To visualize the monoclonal antibodies in the sections, fluorescein-labeled avidin D and 5-bromo-4-chloro-3-indolyl phosphate/nitro blue tetrazolium (BCIP/NBT) are used for the anti-mouse IgG antibodies (panel A, Slow; panel B, Fast; panel C, 71) and the anti-mouse IgM antibodies (panel D, F3), respectively. Labels for fibers containing each MHC isoform: 1, MHCI; 2, MHCIIa; 3, hybrid fibers containing both MHCI and MHCIIa; labels for fibers enzymehistochemically identified: 4, type I; 5, type IIA; 6, type IIC. Scale bar on panel $\mathbf{H}$ indicates $50 \mu \mathrm{m}$ for all panels. 

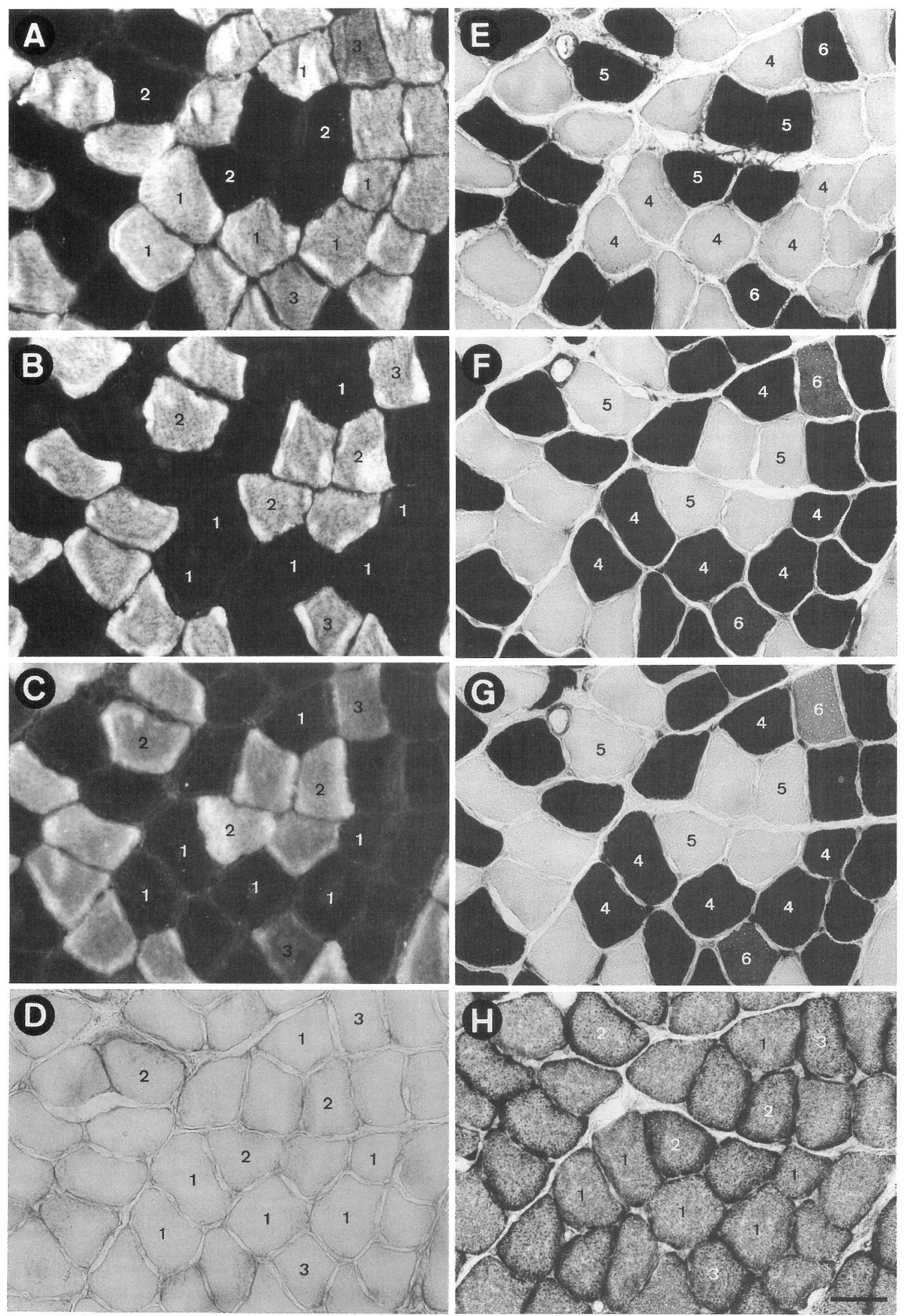

Fig. 1 
ized by the coexistence of both MHCI and MHCIIa in varying ratios [77]. The proportion of different MHC isoforms within a single fiber is uncertain, since the immunohistochemical analysis is not quantitative because of the difference in the sensitivity of each MAb and in the different amplification processes.
The rat plantaris muscle is a typical fast-twitch muscle, and its serial transverse sections are shown in panels A to $\mathrm{H}$ of Fig. 2. It contains four major $\mathrm{MHC}$ isoforms [60]. The isoform proportions are different between the deep and superficial portions of the muscle, and they also change with postnatal development [84]. In the plantaris
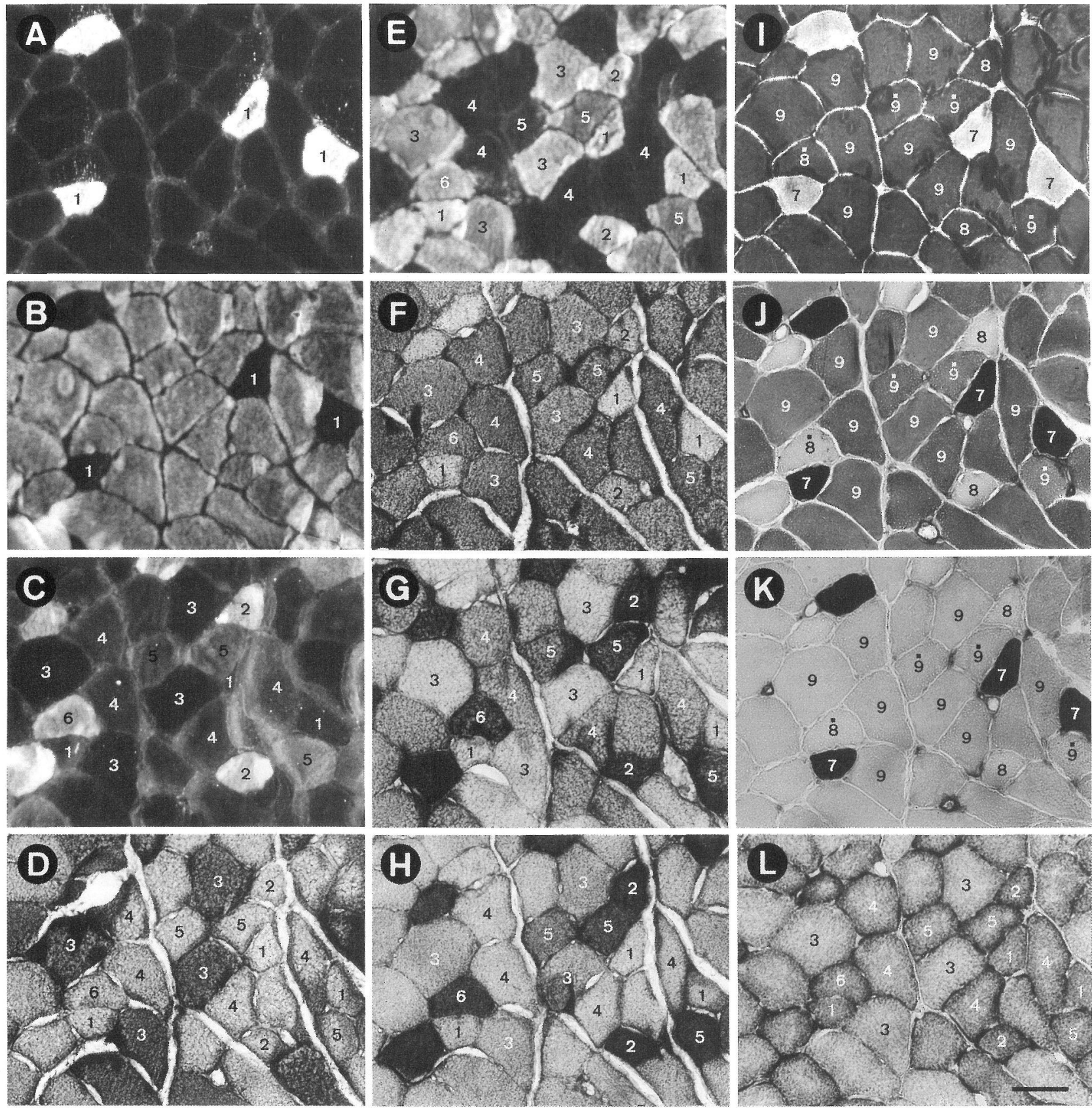

Fig. 2. Serial transverse sections of the plantaris muscle of an 8-week-old rat. The sections are stained with the specific monoclonal antibodies against specific myosin heavy chain (MHC) isoforms (panels $\mathbf{A}-\mathbf{H}$, see Table 1 for specificity) and for ATPase activities following preincubations at pH 10.3 (panel I), 4.5 (panel $\mathbf{J}$ ) and 4.3 (panel $\mathbf{K}$ ) and for SDH activity (panel $\mathbf{L}$ ). To visualize the monoclonal antibodies in the sections, fluorescein-labeled avidin D and 5-bromo-4-chloro-3-indolyl phosphate/nitro blue tetrazolium (BCIP/NBT) are used for anti-mouse IgG antibodies (panel $\mathbf{A}$, Slow; panel $\mathbf{B}$, Fast; panel $\mathbf{C}$, 71; panel $\mathbf{E}, 35$ ) and anti-mouse IgM antibodies (panel $\mathbf{D}, \mathbf{F} 3$; panel $\mathbf{F}$, D9; panel $\mathbf{G}$, N1.551; panel H, A4.1519), respectively. Labels for fibers containing each MHC isoform: 1, MHCI; 2, MHCIIa; 3, MHCIIb; 4, MHCIIx; 5 and 6, hybrid IIxa and IIax fibers containing both MHCIIa and MHCIIx, respectively; labels for fibers enzyme-histochemically identified: 7, type I; 8 (and 8), type IIA; 9 (and 9), type IIB (see text for details). Scale bar on panel $\mathbf{L}$ indicates $50 \mu \mathrm{m}$ for all panels. 
muscle of the adult rat, as shown in panels A and B, about $15 \%$ of the fibers have MHCI in the deep portion (marked as 1), while the proportion of MHCI is less in the superficial portion of the muscle. The fibers containing MHCIIb (marked as 3) or MHCIIx (marked as 4) are predominant, and some fibers have MHCIIa (marked as 2). At present, a MAb specific for MHCIIx alone is not available, and therefore, the fibers containing only the MHCIIx can be identified with the negative staining for MAb 35 (specific for all types of MHCs except MHCIIx, panel E). The inconvenience is that MHCIIx included in the hybrid fibers cannot be precisely detected with only MAb 35 . The combination of several MAbs is necessary for fibertype classifications including MHCIIx fibers.

Four hybrid fibers are shown in Fig. 2. Three fibers marked as 5 are stained weakly with MAb 35 (panel E). They are positive for MAbs Fast (panel B), 71 (panel C), D9 (panel F), N1.551 (panel G), and A4.1519 (panel H), and negative for Slow (panel A) and F3 (panel D). This means that they have MHCIIa in addition to MHCIIx. Another hybrid fiber marked as 6 cannot be verified as having MHCIIx by the staining with MAb 35 (panel E). The positive staining for D9 (panel F) and the negative staining for F3 (panel D) reveal the possibility that this fiber has MHCIIx, probably in a small amount. We decided to name these fibers IIxa (marked as 5) and IIax (marked as 6) according to the proportion of the isoforms within a single fiber. For example, the fiber name "IIxa" means that the percentage of the MHCIIx is higher than that of the MHCIIa in a single fiber, while the fiber name "IIax" means that the percentage of the MHCIIa is higher than that of the MHCIIx in a single fiber.

\section{MHC Isoform and ATPase Histochemistry}

The histochemical ATPase activities of single muscle fibers based on the differential responses to alkali and acid preincubations correspond to their MHC isoform profiles. This correspondence was analyzed in studies combining the immunohistochemistry and/or electrophoresis of either the whole muscle or the single muscle fiber $[45,70$, 77, 78, 81, 93, 94].

Muscle fibers containing MHCI correspond to type I fibers, and muscle fibers containing MHCIIa correspond to type IIA fibers. Type IIB fibers determined by the ATPase staining reaction include MHCIIb and/or MHCIIx, and some modifications of the staining method using ATPase activity have been developed in order to distinguish MHCIIx from MHCIIb [31, 35, 65]. Type IIC fibers, which are active at all pHs throughout alkali to acid ATPase preincubations, correspond to hybrid fibers containing both MHCI and MHCII (mainly MHCIIa) [78]. In general, alkali-ATPase measures the fastest MHC isoform present in the fiber, whereas acidATPase reflects the average ATPase activity associated with the proportion of $\mathrm{MHC}$ isoform types within the fiber [90]. Interspecies differences in the $\mathrm{pH}$ stability of ATPase activity have been noted $[16,17]$.

\section{MHC Isoform and Metabolic Enzyme Histochemistry}

A correlation between metabolic enzyme activities and $\mathrm{MHC}$ isoforms has been reported histochemically [44, 59] and biochemically [22]. The linkage between MHC isoforms and the metabolic properties is supported by their coordinated alterations under muscle fiber-type transformation [25, 44]. However, there is a report that individual motor unit fibers have uniform SDH activity even if they are composed of mixed MHC isoforms [72]. As there is no direct relation between the MHC molecule and metabolic enzyme activity, they may influence each other to achieve the best match for functional demand. Generally, oxidative enzyme activities, i.e., SDH and citrate synthase activities are reported to be in the rank order of MHCIIa $>$ MHCI $>$ MHCIIx $>$ MHCIIb, and glycolytic enzyme activity, i.e., $\alpha$-glycerophosphate dehydrogenase $(\alpha-\mathrm{GPD})$ activity is in the rank order of MHCIIb $>$ MHCIIx $>$ MHCIIa $>$ MHCI [25].

\section{MHC Isoform Analysis by Other Methods}

MHC proteins of muscle homogenates are analyzed with electrophoresis. Four major MHC isoforms in adult skeletal muscle are completely separated [85], and the rank order of migration is MHCI $>$ MHCIIb $>$ MHCIIx $>$ MHCIIa. It should be noted that the electrophoretic mobility shows interspecies differences even within identically labeled isoforms [36]. Single fiber electrophoretic studies support the coexistence of multiple MHC isoforms in individual fibers $[51,93]$. Previous studies have shown that the hybrid fiber is not an exception but exists as a standard [36, 56, 69].

MHC isoforms are also identified at the mRNA level $[12,20,23,42]$. Hybrid fibers are detected as the coexistence of multiple MHC mRNA by in situ hybridization $[12,23]$ and reverse-transcriptase polymerase chain reaction (RT-PCR) directly from single fibers [57]. Combined studies with immuno- or enzyme-histochemistry have confirmed the correspondence of MHC protein and mRNA $[23$, 57]. During muscle fiber-type transformation, however, the mismatch between the content of MHC protein and the expression of mRNA occurs in transform-

Table 2. Adenosine triphosphatase activity

\begin{tabular}{ccccc}
\hline Preincubation & \multicolumn{5}{c}{ Fiber type } \\
\cline { 2 - 5 } $\mathrm{pH}$ & I & IIA & IIB & IIC \\
\hline 10.3 & - & + & + & + \\
4.5 & + & - & + & + \\
4.3 & + & - & - & + \\
\hline
\end{tabular}

The fiber type classification was determined by Brooke and Kaiser [16-18]. +, positive reaction; -, no reaction. 
ing fibers [2, 12]. The distribution of MHC mRNA along the fiber length is also reported to be nonuniform [57].

\section{Classical Identification of Muscle Fiber}

Based on the differences in the ATPase activity following preincubations at different $\mathrm{pHs}$, skeletal muscle fibers are classified into two major types; acid-stable and alkali-labile type I (slow-twitch) and acid-labile and alkalistable type II (fast-twitch) [34]. Type II fibers are further subclassified into type IIA and type IIB according to their ATPase stabilities in the acid range; type IIA fibers become ATPase-negative prior to type IIB fibers when the acidity of the preincubation medium increases [16-18] (Table 2).

Figure 1 shows the correspondence between the expression of MHC isoforms and the staining pattern of ATPase in the rat soleus muscle. The fibers containing MHCI marked as 1 (panels A to D) are negative at $\mathrm{pH} 10.3$ (panel E) and positive at pH 4.5 (panel F) and 4.3 (panel $\mathrm{G})$, and thus correspond to the type I fibers marked as 4 in panels E to G. The fibers containing MHCIIa marked as 2 are positive at $\mathrm{pH} 10.3$ and negative at $\mathrm{pH} 4.5$ and 4.3, corresponding to the type IIA fibers marked as 5 in panels $\mathrm{E}$ to $\mathrm{G}$. The hybrid fibers containing both MHCI and MHCIIa marked as 3 are positive at all pHs examined, and correspond to the type IIC fibers marked as 6 in panels E to G. Type IIC fibers are exclusively found during regeneration processes including embryonic and neonatal stages. The SDH activities in the fibers containing MHCIIa (type IIA) and both MHCI and MHCIIa (type IIC) are higher than those in the fibers containing only MHCI (type I) (panel H).

In the plantaris muscle, the hybrid fibers are classified with ATPase and SDH staining; MHCIIax fibers determined by immunohistochemistry (panels A to $\mathrm{H}$ of Fig. 2) are classified as type IIA, marked as 8 (panels I to $\mathrm{K}$ ) and MHCIIxa fibers are classified as type IIB, marked as $\dot{9}$ (panels I to K). Pure MHCIIb (marked as 3) and MHCIIx (marked as 4) fibers cannot be distinguished with ATPase staining, although SDH activity is higher in MHCIIx fibers than in MHCIIb fibers (panel L) as reported previously $[22,40,67]$.

Another commonly used classification of skeletal muscle fibers is based on the differences in the energy utilization and metabolic demand $[54,55]$. Oxidative enzyme activities, i.e., SDH and nicotinamide adenine dinucleotide dehydrogenase activities are related to the mitochondrial aerobic pathway for oxidative energy supply, whereas the glycolytic energy supply pathway is associated with the enzyme activity, i.e., $\alpha$-GPD activity. By combining the ATPase-based and oxidative and glycolytic enzyme-based classification, skeletal muscle fibers are categorized into three types; fast-twitch oxidative glycolytic (FOG), fast-twitch glycolytic (FG), and slow-twitch oxidative (SO) $[4,54]$.

MHC isoforms of skeletal muscle fibers correspond to certain types of muscle fibers determined by the ordinary enzyme histochemical classification [37, 39, 56, 76, 78, 79] as shown in Figs. 1 and 2. Types IIA (FOG), IIB (FG), and I (SO) fibers defined by enzyme histochemistry correspond to types MHCIIa, MHCIIb, and MHCI fibers, respectively. However, the variation of MHC isoforms is far more than we would expect from the results obtained by enzyme histochemistry. Thus, multiple MHC isoforms can coexist in a single muscle fiber $[1,11,77]$, an aspect which further increases the diversity of muscle fiber properties. These hybrid fibers are found even in mature skeletal muscles under normal conditions [36], and they become more prominent during muscle fiber-type transformation [56].

The immunohistochemical method using monoclonal antibodies which are specific for different MHC isoforms provides useful information regarding muscle fiber-type plasticity which could not be obtained by the earlier enzyme histochemical methods.

\section{MHC Isoform Transformation}

The composition of MHC isoforms in skeletal muscle is dynamic, not static. The $\mathrm{MHC}$ isoforms are adaptive to the physiological changes such as development $[24,30,43$, $50,58,63,96]$, aging $[46,66]$, increased $[3,9,27,64,82]$ or decreased [10,51,88,89] neuromuscular activity, hormones [32, 47, 50,61], and other factors [73]. For example, all fibers in rat muscles have MHCemb at the perinatal stage, and then they differentiate to coexpress MHCneo and/or MHCI [50, 63]. The growth-related transformation of fast-to-slow and slow-to-fast MHC isoforms is observed in the slow- and fast-twitch muscles, respectively, and fibers containing MHCemb and MHCneo disappear during the first month of life $[30,58]$. In contrast, the transformation of the fast-to-slow MHC isoform generally occurs during the aging process $[44,46]$. For example, fibers containing MHCIIa, which are about $20 \%$ of the slow-twitch soleus muscle of the adult rat, disappear at the latest stage by transforming from MHCIIa to MHCI. Even in the fast-twitch muscle, fibers containing the fastest type (MHCIIb) are progressively transformed to those containing the second fastest type (MHCIIx) during aging. Neuromuscular activity is one of the important factors which affect MHC isoform profiles; increased neuromuscular activity and mechanical overloading for muscle cause the transformation in the fast-to-slow direction $[3,9,64]$, whereas decreased activity and unloading result in the slow-to-fast transformation [10, 51, 88, 89]. Hyperthyroidism causes the transformation in the slowto-fast direction, and hypothyroidism causes the transformation in the opposite direction [32, 50].

Muscle fiber-type transformation occurs in order to provide the contractile or metabolic property most adequate for the new condition. The MHC isoform profile in a single fiber is a good marker of muscle fibertype transformation, and immunohistochemistry can 
easily detect it. MHC isoform transformation has been reported to occur as a sequential conversion from MHCI $\leftrightarrow$ MHCIIa $\leftrightarrow$ MHCIIx $\leftrightarrow$ MHCIIb [5, 81, 93]. However, careful immunohistochemical observation revealed that a "jump" from MHCI to MHCIIx, for example, can occur under special conditions [87]. The proportion of the hybrid fibers apparently increases under muscle fibertype transformation. Up to four different isoforms are found to be coexpressed in transforming fibers [93]. An immunogold electron microscopic study revealed that specific MHC isoforms are segregated in some distinct populations of myofibrils within individual fibers [29]. In isolated myosin thick filaments, the coexistence of two MHC isoforms has been observed [92].

Immunohistochemistry can qualitatively identify the MHC profile of hybrid fibers by combining several MAbs. At present, the variety of specific antibodies for MHC isoforms is small, which limits the usage of the doublelabeling technique on the same preparation. An investigator must prepare as many serial sections as the number of MAbs to be used, and, therefore, many sections including the same fiber are needed. This is undesirable for analyzing MHC proteins, which have a nonuniform distribution along the longitudinal axis of the muscle fiber. Another problem is the quantification of the MHC isoform content on the histological preparation. There are some ongoing trials to quantify the immunoreactivity of individual muscle fibers $[48,53]$. The development of easy and reliable quantification methods will be useful for immunohistochemical analyses of hybrid fibers. In addition, there may well be MHC isoforms as yet unidentified and histochemically undetectable.

Although muscle fiber-type transformation is one of the major topics in bioscience, the regulatory mechanism of the gene expression involved is not yet clear. The transformation is known to occur not only in MHC proteins but also in other proteins such as troponin and myosin light chain [69], and there may be a mechanism of coupling in the regulatory elements. Detailed histochemical observations of the transformation process in response to various stimuli and environmental changes are necessary. The genetic regulation in multinuclear cells such as skeletal muscle fibers is also a problem; whether a single nucleus produces mRNA of a single isoform or multiple isoforms. Does the MHC protein produced remain within the nuclear domain of the nucleus which has expressed its mRNA [49]? Does the transformation occur simultaneously along the longitudinal axis of a muscle fiber [52]? If not, where does it start, and how is it coordinated along the fiber's full length? Although mechanisms regulating muscle fiber-type diversity have been extensively investigated [33], advanced immunohistochemical approaches will help to answer these remaining questions.

\section{Conclusion}

We have described the immunohistochemical methods used for the classification of muscle fiber-types and their utility, especially for detecting hybrid fibers using different monoclonal antibodies against specific MHC isoforms. These methods are essential for studying how specific kinds and amounts of proteins are synthesized and expressed in single fibers. Immunohistochemical data will help to clarify the dynamic nature of muscle fibers and the mechanism of muscle fiber-type transformation.

\section{Acknowledgments}

We thank Dr. S. Schiaffino (University of Padova, Italy) for providing us with the monoclonal antibodies 71 , F3, 35, and D9. The monoclonal antibodies N1.551 and A4.1519 developed by Dr. H. Blau were obtained from the Developmental Studies Hybridoma Bank maintained by the Department of Pharmacology and Molecular Sciences, Johns Hopkins University School of Medicine and the Department of Biology, University of Iowa.

\section{References}

1. Aigner, S., Gohlsch, B., Hämäläinen, N., Staron, R. S., Uber, A., Wehrle, U. and Pette, D.: Fast myosin heavy chain diversity in skeletal muscles of the rabbit: heavy chain IId, not IIb predominates. Eur. J. Biochem. 211; 367-372, 1993.

2. Andersen, J. L. and Schiaffino, S.: Mismatch between myosin heavy chain mRNA and protein distribution in human skeletal muscle fibers. Am. J. Physiol. 272; C1881-C1889, 1997.

3. Andersen, J. L., Mohr, T., Biering-Sorensen, F., Galbo, H. and Kjær, M.: Myosin heavy chain isoform transformation in single fibres from $\mathrm{m}$. vastus lateralis in spinal cord injured individuals: effects of long-term functional electrical stimulation (FES). Pflügers Arch. 431; 513-518, 1996.

4. Armstrong, R. B. and Phelps, R. O.: Muscle fiber type composition of the rat hindlimb. Am. J. Anat. 171;259-272, 1984.

5. Ausoni, S., Gorza, L., Schiaffino, S., Gundersen, K. and Lømo, T.: Expression of myosin heavy chain isoforms in stimulated fast and slow rat muscles. J. Neurosci. 10; 153-160, 1990.

6. Bandman, E.: Contractile protein isoforms in muscle development. Dev. Biol. 154; 273-283, 1992.

7. Bar, A. and Pette, D.: Three fast myosin heavy chains in adult rat skeletal muscle. Fed. Eur. Biochem. Soc. Lett. 235; 153$155,1988$.

8. Bárány, M.: ATPase activity of myosin correlated with speed of muscle shortening. J. Gen. Physiol. 50; 197-218, 1967.

9. Baumann, H., Jäggi, M., Soland, F., Howald, H. and Schaub, M. C.: Exercise training induces transitions of myosin isoform subunits within histochemically typed human muscle fibres. Pflügers Arch. 409; 349-360, 1987.

10. Bigard, A.X., Serrurier, B., Merino, D., Lienhard, F., Berthelot, M. and Guezennec, C. Y.: Myosin heavy chain composition of regenerated soleus muscles during hindlimb suspension. Acta Physiol. Scand. 161; 23-30, 1997.

11. Biral, D., Betto, R., Danieli-Betto, D. and Salviati, G.: Myosin heavy chain composition of single fibres from normal human muscle. Biochem. J. 250; 307-308, 1988.

12. Bodine, S. C. and Pierotti, D. J.: Myosin heavy chain mRNA and protein expression in single fibers of the rat soleus following reinnervation. Neurosci. Lett. 215; 13-16, 1996.

13. Bottinelli, R., Schiaffino, S. and Reggiani, C.: Force-velocity relations and myosin heavy chain isoform compositions of skinned fibres from rat skeletal muscle. J. Physiol. 437; 655- 
$672,1991$.

14. Bottinelli, R., Betto, R., Schiaffino, S. and Reggiani, C.: Maximum shortening velocity and coexistence of myosin heavy chain isoforms in single skinned fast fibres of rat skeletal muscle. J. Muscle Res. Cell Motil. 15; 413-419, 1994.

15. Bottinelli, R., Canepari, M., Reggiani, C. and Stienen, G. J.: Myofibrillar ATPase activity during isometric contraction and isomyosin composition in rat single skinned muscle fibres. $J$. Physiol. 481; 663-675, 1994.

16. Brooke, M. H. and Kaiser, K. K.: Muscle fiber types: how many and what kind? Arch. Neurol. 23; 369-379, 1970.

17. Brooke, M. H. and Kaiser, K. K.: Three "myosin adenosine triphosphatase" systems: the nature of their pH lability and sulfhydryl dependence. J. Histochem. Cytochem. 18; 670-672, 1970.

18. Brooke, M.H. and Kaiser, K. K.: Three human myosin ATPase systems and their importance in muscle pathology. Neurology 20; 404-405, 1970.

19. Brueckner, J. K., Itkis, O. and Porter, J. D.: Spatial and temporal patterns of myosin heavy chain expression in developing rat extraocular muscle. J. Muscle Res. Cell Motil. 17; 297-312, 1996.

20. Camoretti-Mercado, B., Qin, Y., Jakovcic, S., Salazar-Grueso, E. and Zak, R.: Developmental shift of myosin heavy chain mRNA expression due to neural factor(s) and muscle activity. Am. J. Physiol. 271; C1350-C1357, 1996.

21. Danieli-Betto, D., Zerbato, E. and Betto, R.: Type I, IIa and IIb myosin heavy chain electrophoretic analysis of rat muscle fibres. Biochem. Biophys. Res. Commun. 138; 981-987, 1986.

22. Delp, M. D. and Duan, C.: Composition and size of type I, IIA, IID/X, and IIB fibers and citrate synthase activity of rat muscle. J. Appl. Physiol. 80; 261-270, 1996.

23. DeNardi, C., Ausoni, S., Moretti, P., Gorza, L., Velleca, M., Buckingham, M. and Schiaffino, S.: Type 2X-myosin heavy chain is coded by a muscle fiber type-specific and developmentally regulated gene. J. Cell Biol. 123; 823-835, 1993.

24. Dhoot, G. K.: Selective synthesis and degradation of slow skeletal myosin heavy chains in developing muscle fibers. Muscle Nerve 9; 155-164, 1986.

25. Dunn, S. E. and Michel, R. N.: Coordinated expression of myosin heavy chain isoforms and metabolic enzymes within overloaded rat muscle fibers. Am. J. Physiol. 273; C371-C383, 1997.

26. Fauteck, S. P. and Kandarian, S. C.: Sensitive detection of myosin heavy chain composition in skeletal muscle under different loading conditions. Am. J. Physiol. 268; C419-C424, 1995.

27. Galler, S., Hilber, K. and Pette, D.: Stretch activation and myosin heavy chain isoforms of rat, rabbit and human skeletal muscle fibres. J. Muscle Res. Cell Motil. 18; 441-448, 1997.

28. Galler, S., Hilber, K., Gohlsch, B. and Pette, D.: Two functionally distinct myosin heavy chain isoforms in slow skeletal muscle fibres. FEBS Lett. 410; 150-152, 1997.

29. Gauthier, G. F.: Differential distribution of myosin isoforms among the myofibrils of individual developing muscle fibers. $J$. Cell Biol. 110; 693-701, 1990.

30. Gondret, F., Lefaucheur, L., D'Albis, A. and Bonneau, M.: Myosin isoform transitions in four rabbit muscles during postnatal growth. J. Muscle Res. Cell Motil. 17; 657-668, 1996.

31. Gorza, L.: Identification of a novel type 2 fiber population in mammalian skeletal muscle by combined use of histochemical myosin ATPase and anti-myosin monoclonal antibodies. $J$. Histochem. Cytochem. 38; 257-265, 1990.

32. Gosselin, L. E., Zhan, W. -Z. and Sieck, G. C.: Hypothyroidmediated changes in adult rat diaphragm muscle contractile properties and MHC isoform expression. J. Appl. Physiol. 80;
1934-1939, 1996.

33. Gunning, P. and Hardeman, E.: Multiple mechanisms regulate muscle fiber diversity. FASEB J. 5; 3064-3070, 1991.

34. Guth, L. and Samaha, F. J.: Qualitative differences between actomyosin ATPase of slow and fast mammalian muscle. Exp. Neurol. 25; 138-152, 1969.

35. Hämäläinen, N. and Pette, D.: The histochemical profiles of fast fiber types IIB, IID, and IIA in skeletal muscles of mouse, rat, and rabbit. J. Histochem. Cytochem. 41; 733-743, 1993.

36. Hämäläinen, N. and Pette, D.: Patterns of myosin isoforms in mammalian skeletal muscle fibres. Microsc. Res. Tech. 30; 381-389, 1995.

37. Havenith, M. G., Visser, R., Schrijvers-van Schendel, J. M. C. and Bosman, F. T.: Muscle fiber typing in routinely processed skeletal muscle with monoclonal antibodies. Histochemistry 93; 497-499, 1990.

38. Hilber, K., Galler, S. and Pette, D.: Functional differences of myosin heavy-chain isoforms in skeletal muscle. Naturwissenschaften 84; 201-204, 1997.

39. Ishihara, A., Itoh, K., Oishi, Y., Itoh, M., Hirofuji, C. and Hayashi, H.: Effects of hypobaric hypoxia on histochemical fibre-type composition and myosin heavy chain isoform component in the rat soleus muscle. Pflügers Arch. 429; 601-606, 1995.

40. Ishihara, A., Hori, A., Roy, R. R., Oishi, Y., Talmadge, R. J., Ohira, Y., Kobayashi, S. and Edgerton, V. R.: Perineal muscles and their innervation: metabolic and functional significance of the motor unit. Acta Anat. 159; 156-166, 1997.

41. Izumo, S., Nadal-Ginard, B. and Mahdavi, V.: All members of the MHC multigene family respond to thyroid hormone in a highly tissue-specific manner. Science 231; 597-600, 1986.

42. Jänkälä, H., Harjola, V. -P., Petersen, N. E. and Härkönen, M.: Myosin heavy chain mRNA transform to faster isoforms in immobilized skeletal muscle: a quantitative PCR study. $J$. Appl. Physiol. 82; 977-982, 1997.

43. Kucera, J. and Walro, J. M.: Transient expression of a slowtonic MHC isoform by extrafusal fibers in the developing rat. Anat. Embryol. 188; 409-418, 1993.

44. Larsson, L., Ansved, T., Edström, L., Gorza, L. and Schiaffino, S.: Effects of age on physiological, immunohistochemical and biochemical properties of fast-twitch single motor units in the rat. J. Physiol. 443; 257-275, 1991.

45. Larsson, L., Edström, L., Lindegren, B., Gorza, L. and Schiaffino, S.: MHC composition and enzyme-histochemical and physiological properties of a novel fast-twitch motor unit type. Am. J. Physiol. 261; C93-C101, 1991.

46. Larsson, L., Li, X. and Frontera, W. R.: Effects of aging on shortening velocity and myosin isoform composition in single human skeletal muscle cells. Am. J. Physiol. 272; C638-C649, 1997.

47. Mahdavi, V., Izumo, S. and Nadal-Ginard, B.: Developmental and hormonal regulation of sarcomeric myosin heavy chain gene family. Circ. Res. 60; 804-814, 1987.

48. Manabe, N., Azuma, Y., Furuya, Y., Kuramitsu, K., Nagano, N. and Miyamoto, H.: Immunohistochemical microquantification of fast-myosin in frozen histological sections of mammalian skeletal muscles. J. Animal Sci. 73; 88-95, 1995.

49. Mascarello, F., Veggetti, A., Cerpene, E. and Rowlerson, A.: An immunohistochemical study of the middle ear muscles of some carnivores and primates, with special reference to the IIM and slow-tonic fibre types. J. Anat. 137; 95-108, 1983.

50. Narusawa, M., Fitzsimons, R. B., Izumo, S., Nadal-Ginard, B., Rubinstein, N. A. and Kelly, A. M.: Slow myosin in developing rat skeletal muscle. J. Cell Biol. 104; 447-459, 1987.

51. Oishi, Y., Ishihara, A., Yamamoto, H. and Miyamoto, E.: Hindlimb suspension induces the expression of multiple myosin 
heavy chain isoforms in single fibres of the rat soleus muscle. Acta Physiol. Scand. 162, 127-134, 1998.

52. Pavlath, G. K., Rich, K., Webster, S. G. and Blau, H. M.: Localization of muscle gene products in nuclear domains. Nature 337; 570-573, 1989.

53. Pereira Sant'Ana, J. A., Ennion, S., Sargeant, A. J., Moorman, A.F. and Goldspink, G.: Comparison of the molecular, antigenic and ATPase determinants of fast myosin heavy chains in rat and human: a single-fibre study. Pflügers Arch. 435; 151-163, 1997.

54. Peter, J. B., Barnard, R. J., Edgerton, V. R., Gillespie, C. A. and Stempel, K. E.: Metabolic profiles of three fiber types of skeletal muscle in guinea pigs and rabbits. Biochemistry 11; 2627-2633, 1972.

55. Pette, D. and Spamer, C.: Metabolic properties of muscle fibers. Fed. Proc. 45; 2910-2914, 1986.

56. Pette, D. and Staron, R. S.: Mammalian skeletal muscle fiber type transitions. Int. Rev. Cytol. 170; 143-223, 1997.

57. Peuker, H. and Pette, D.: Quantitative analyses of myosin heavy-chain mRNA and protein isoforms in single fibers reveal a pronounced fiber heterogeneity in normal rabbit muscles. Eur. J. Biochem. 247; 30-36, 1997.

58. Picquet, F., Stevens, L., Butler-Brown, G. S. and Mounier, Y.: Contractile properties and myosin heavy chain composition of newborn rat soleus muscles at different stages of postnatal development. J. Muscle Res. Cell Motil. 18; 71-79, 1997.

59. Reichmann, H. and Pette, D.: A comparative microphotometric study of succinate dehydrogenase activity levels in type I, IIA and IIB fibres of mammalian and human muscles. Histochemistry 74; 27-41, 1982.

60. Roy, R. R., Talmadge, R. J., Fox, K., Lee, M., Ishihara, A. and Edgerton, V. R.: Modulation of MHC isoforms in functionally overloaded and exercised rat plantaris fibers. J. Appl. Physiol. 83; 280-290, 1997.

61. Roy, R. R., Tri, C., Grossman, E. J., Talmadge, R. J., Grindeland, R. E., Mukku, V. R. and Edgerton, V. R.: IGF-I, growth hormone, and/or exercise effects on non-weight-bearing soleus of hypophysectomized rats. J. Appl. Physiol. 81; 302311, 1996.

62. Rushbrook, J. I., Weiss, C., Ko, K., Feuerman, M. H., Carleton, S., Ing, A. and Jacoby, J.: Identification of alphacardiac myosin heavy chain mRNA and protein in extraocular muscle of the adult rabbit. J. Muscle Res. Cell Motil. 15; 505515, 1994.

63. Russell, S. D., Cambon, N. A. and Whalen, R. G.: Two types of neonatal-to-adult fast myosin heavy chain transitions in rat hindlimb muscle fibers. Dev. Biol. 157; 359-370, 1993.

64. Salmons, S.: Exercise, stimulation and type transformation of skeletal muscle. Int. J. Sports Med. 15; 136-141, 1994.

65. Sant'Ana Pereira, J.A. A., de Haan, A., Wessels, A., Moorman, A.F. M. and Sargeant, A. J.: The mATPase histochemical profile of rat type IIX fibres: correlation with myosin heavy chain immunolabelling. Histochem. J. 27; 715$722,1995$.

66. Savolainen, J. and Vornanen, M.: Myosin heavy chains in skeletal muscles of the common shrew (Sorex araneus) : absence of a slow isoform and transitions of fast isoforms with ageing. Acta Physiol. Scand. 155, 233-239, 1995.

67. Sawchak, J. A., Leung, B. and Shafiq, S. A.: Evidence for new isoform of fast myosin heavy chain in rat skeletal muscle. Muscle Nerve 15; 1349-1353, 1992.

68. Schiaffino, S. and Reggiani, C.: Myosin isoforms in mammalian skeletal muscle. J. Appl. Physiol. 77; 493-501, 1994.

69. Schiaffino, S. and Reggiani, C.: Molecular diversity of myofibrillar proteins: gene regulation and functional significance. Physiol. Rev. 76; 371-423, 1996.
70. Schiaffino, S., Gorza, L., Sartore, S., Saggin, L., Ausoni, S., Vianello, M., Gundersen, K. and Lømo, T.: Three myosin heavy chain isoforms in type 2 skeletal muscle fibres. J. Muscle Res. Cell Motil. 10; 197-205, 1989.

71. Schmitt, T. L. and Pette, D.: Fiber type-specific distribution of parvalbumin in rabbit skeletal muscle: a quantitative microbiochemical and immunohistochemical study. Histochemistry 96; 459-465, 1991.

72. Sieck, G. C., Fournier, M., Prakash, Y. S. and Blanco, C. E.: Myosin phenotype and SDH enzyme variability among motor unit fibers. J. Appl. Physiol. 80; 2179-2189, 1996.

73. Snoj-Cvetko, E., Smerdu, V., Sketelj, J., Dolenc, I., D’Allbis, A., Janmot, C. and Erzen, I.: Adaptive range of myosin heavy chain expression in regenerating soleus is broader than in mature muscle. J. Muscle Res. Cell Motil. 17; 401-409, 1996.

74. Sosnicki, A. A., Lutz, G. J., Rome, C. and Goble, D. O.: Histochemical and metabolic determination of fiber types in chemically skinned single equine skeletal muscle fibers. $J$. Histochem. Cytochem. 37; 1731-1738, 1989.

75. Spudich, J. A.: How molecular motors work. Nature 372; 515-518, 1994.

76. Stål, P., Eriksson, P. -O., Schiaffino, S., Butler-Brown, G. S. and Thornell, L. -E.: Differences in myosin composition between human oro-facial, masticatoy and limb muscles: enzyme-, immuno- and biochemical studies. J. Muscle Res. Cell Motil. 15; 517-534, 1994.

77. Staron, R. S.: Correlation between myofibrillar ATPase activity and myosin heavy chain composition in single human muscle fibers. Histochemistry 96; 21-24, 1991.

78. Staron, R.S. and Pette, D.: Correlation between myofibrillar ATPase activity and myosin heavy chain composition in rabbit muscle fibers. Histochemistry 86; 19-23, 1986.

79. Staron, R. S. and Pette, D.: The multiplicity of combinations of myosin light chains and heavy chains in histochemically typed single fibers. Biochem. J. 243; 695-699, 1987.

80. Staron, R.S. and Pette, D.: Nonuniform myosin expression along single fibers of chronically stimulated and contralateral rabbit tibialis anterior muscles. Pflügers Arch. 409; 67-73, 1987.

81. Staron, R. S. and Pette, D.: The continuum of pure and hybrid myosin heavy chain-based fibre types in rat skeletal muscle. Histochemistry 100; 149-153, 1993.

82. Staron, R. S., Gohlsch, B. and Pette, D.: Myosin polymorphism in single fibers of chronically stimulated rabbit fast-twitch muscle. Pflügers Arch. 408; 444-450, 1987.

83. Sugiura, T., Matoba, H., Miyata, H., Kawai, Y. and Murakami, N.: Myosin heavy chain isoform transition in ageing fast and slow muscles of the rat. Acta Physiol. Scand. 144; 419-423, 1992.

84. Sullivan, V. K., Powers, S. K., Criswell, D. S., Tumer, N., Larochelle, J.S. and Lowenthal, D.: Myosin heavy chain composition in young and old rat skeletal muscle: effects of endurance exercise. J. Appl. Physiol. 78; 2115-2120, 1995.

85. Talmadge, R. J. and Roy, R. R.: Electrophoretic separation of rat skeletal muscle myosin heavy-chain isoforms. J. Appl. Physiol. 75; 2337-2340, 1993.

86. Talmadge, R. J., Roy, R. R. and Edgerton, V. R.: Muscle fiber types and function. Curr. Opin. Rheumatol. 5; 695-705, 1993.

87. Talmadge, R. J., Roy, R. R. and Edgerton, V. R.: Prominence of myosin heavy chain hybrid fibers in soleus muscle of spinal cord-transected rats. J. Appl. Physiol. 78; 1256-1265, 1995.

88. Talmadge, R. J., Roy, R. R. and Edgerton, V. R.: Distribution of myosin heavy chain isoforms in non-weight-bearing rat soleus muscle fibers. J. Appl. Physiol. 81; 2540-2546, 1996.

89. Talmadge, R. J., Roy, R. R. and Edgerton, V. R.: Myosin heavy chain profile of cat soleus following chronic reduced ac- 
tivity or inactivity. Muscle Nerve 19; 980-988, 1996.

90. Talmadge, R. J., Roy, R. R., Jiang, B. and Edgerton, V. R.: Myofibrillar ATPase activity of feline muscle fibers expressing slow and fast myosin heavy chains. J. Histochem. Cytochem. 43; 811-819, 1995.

91. Talmadge, R. J., Roy, R. R., Chalmers, G. R. and Edgerton, V. R.: MHC and sarcoplasmic reticulum protein isoforms in functionally overloaded cat plantaris muscle fibers. J. Appl. Physiol. 80; 1296-1303, 1996.

92. Taylor, L. D. and Bandman, E.: Distribution of fast myosin heavy chain isoforms in thick filaments of developing chicken pectoral muscle. J. Cell Biol. 108; 533-542, 1989.

93. Termin, A., Staron, R. S. and Pette, D.: Changes in myosin heavy chain isoforms during chronic low-frequency stimulation of rat fast hindlimb muscles. A single-fiber study. Eur. J. Biochem. 186; 749-754, 1989.

94. Termin, A., Staron, R. S. and Pette, D.: Myosin heavy chain isoforms in histochemically defined fiber types of rat muscle. Histochemistry 92; 453-457, 1989.

95. Zhang, K. -M., Hu, P., Wang, S. -W., Wright, L. D., Wechsler, A. S., Spratt, J. A. and Briggs, F. N.: Fast- and slow-twitch isoforms (SERCA1 and SERCA2a) of sarcoplasmic reticulum Ca-ATPase are expressed simultaneously in chronically stimulated muscle fibers. Pflügers Arch. 433; 766-772, 1997.

96. Zhang, Y., Sher, J. H., Leung, B. and Shafiq, S. A.: An immunocytochemical study of type I muscle fibres in developing human skeletal muscles. J. Neurol. Sci. 80; 1-12, 1987. 